\title{
Allergic form of Meadow's syndrome (Munchausen by proxy)
}

\author{
J O WARNER AND M J HATHAWAY \\ Allergy Clinic, St Mary's Hospital, London
}

SUMMARY We present 17 children from 11 families with the allergic form of Meadow's syndrome. In all cases their mothers believed that they had severe disease due to allergies-in 16 cases to foods and in one to house dust mite. The maternal obsession with allergen avoidance resulted in bizarre diets and life styles. Most mothers were articulate and middle class, and many had marital problems (three single parents). They had a limpet-like attachment to their child and insisted on many medical consultations. Management proved very difficult and despite careful exclusion of allergic disease, many remained on diets and failed allergy clinic follow up. In most cases the obsession with allergy had been initiated by doctors.

'Munchausen's syndrome by proxy', has become the rather cumbersome label attached to children presented by their mothers with a variety of fabricated disorders resulting in extensive unnecessary medical investigations. The characterisation of this condition by Meadow ${ }^{1}$ has now led to the adoption of the author's name in the eponymous labelling of the condition. A recent leading article declared that, 'it is only the lack of particular knowledge of the medical system that prevents more florid development of Meadow's syndrome'. ${ }^{2}$

Food allergy and intolerance has captivated the public imagination over the past two years and extensive airings in the 'mass media' have exposed the public to a large amount of information and misinformation on the hazards of foods. It is, therefore, no surprise that children are now being seen in paediatric clinics with problems that mothers present as caused by food allergy. In an attempt to alert paediatricians to this 'allergic form of Meadow's syndrome', we have gathered details of 17 cases who have presented at St Mary's Paediatric Allergy Clinic.

\section{Methods}

For many decades the Allergy Clinic at St Mary's Hospital has attracted vast numbers of children for investigation of allergic disorders. Four years ago a distinct paediatric allergy session was established and since then approximately 1600 children have attended. Of these 301 have received detailed dietetic assessment for presumed food allergy or intolerance, and 16 of the 17 children presented in this paper are among this number. The remaining child was an extreme example of maternal obsession with aero-allergens, which was an additional feature in two children (siblings) with apparent food related symptoms.

Initial assessment of patients consisted of a full medical history, physical examination, prick skin testing to common inhalant and ingestant allergens including those suggested by history. Where considered appropriate blood samples were taken for total $\mathrm{IgE}$ and $\mathrm{E}$ antibodies by radioallergosorbent test (RAST).

Exclusion diets and challenge. The diets were carefully evaluated by $\mathrm{MJH}$, a paediatric dietician. Inconsistencies in the diets were often a strong indication that the reactions were not genuine. The exclusion diets were rationalised using the diagnostic regimen discussed in a previous paper by us. ${ }^{3}$ Excluded foods were reintroduced at home, individually at one week intervals. The children were then challenged again with foods identified by the mother as causing problems, either on an open or a double blind basis, during a period of inpatient observation. The inpatient assessment included an attempt to observe the mother and child interactions by discreet observation. Where possible a social worker or child psychiatrist, or both took part in the 
assessment. Invariably the mother stayed in hospital with the child, but it was rarely possible to introduce a psychiatrist.

Criteria for diagnosis of food allergy or intolerance. Food intolerance implies a reproducible adverse reaction to a food that occurs even when administered double blind. Food allergy can only be diagnosed if the reaction is associated with evidence of an abnormal immunological reaction to the food. Our criteria for making a positive diagnosis were either:

(1) A consistent reaction from history and open challenge at home associated with a positive skin test or RAST, or both to the same food, or

(2) A consistent reaction from history, open challenge at home and inpatient challenge with no response to control challenge.

In many children presenting at the clinic it was possible to establish a genuine reaction to foods and dietary exclusion resulted in improvement of the condition. Where no food intolerance or allergy could be shown most parents readily accepted the diagnosis and reintroduced normal diets without problems. The 16 cases of 'pseudo-intolerance' presented in this paper created enormous difficulties because although food intolerance could not be established, the mothers refused to accept the opinion and maintained the diets against advice. The parental obsession with diagnosis was very abnormal and was adversely affecting their child's life. There were some additional cases where a diagnosis of food intolerance could not be established, but they are not presented either because the diagnosis was still uncertain or the parental attitudes did not adversely affect the children.

\section{Results}

The Table outlines the essential features of the cases. Nine of the 17 children were presented with behaviour problems associated with so called hyperactivity. One child had nausea and abdominal pains after certain foods and one recurrent coughs, rhinitis, and fevers.

Case 13 was a mother with Meniere's disease who had decided as a result of extensive reading that she and five of her 6 children had food related problems. For simplicity she is presented in the Table rather than the children. Her five children had a variety of apparent food precipitated problems, including diarrhoea in all five, nocturnal enuresis in two, abdominal pains in one, and recurrent urinary infections in one. The foods considered to produce problems included milk in all; gluten in two; and variously cheese, chocolate, coffee, tea, and several meats. Two of the five children had positive skin tests to inhalant, but not ingestant allergens, and both had mild atopic problems (one asthma and one hayfever). There was no evidence of food related disease in any of the children. Numerous lengthy consultations were required to eventually persuade the mother to relax the children's diet. The mother, herself, remained convinced that diet controlled her Meniere's disease, but dietetic assessment showed gross inconsistencies in the diet.

Case 12 was a tall and extremely healthy looking girl with a single mother. She had mild perennial allergic rhinitis due to house dust mite sensitivity. The half hearted recommendations on house mite avoidance measures made by one of us (JOW) resulted in the girl's life being made a misery. The family moved house twice and she changed schools three times within a very short period. Her mother was convinced that her daughter was extremely ill and debarred her repeatedly from school. Her exchange of correspondence with the Allergy Clinic and the local housing and education authorities was unprecedented. The mother eventually had a 'nervous breakdown', diagnosed as schizophrenia, which required hospital admission. During this period her daughter was fostered and thrived without problems.

Cases 10 and 11 were also submitted to extreme aero-allergen avoidance. This included their mother insisting on them sleeping on the back of an upturned wardrobe, wrapped in toilet paper and silver foil rather than blankets. They also were submitted to dietary restrictions. Because of repeated school absences the children were temporarily taken into care, but they subsequently returned to their mother and have moved away from London. Their single mother was extremely disturbed, but it was never possible to secure any psychiatric attention for her. Having been confronted with the evidence that her children did not have food allergy, she created havoc in an outpatient waiting room and had to be threatened with police intervention before she would leave. She has subsequently threatened legal action for negligence in making her son very ill in hospital, although he was obviously healthy throughout the admission.

Case 9 , a boy with genuine petit mal, had unfortunately remained undiagnosed and untreated for many years. By the time an EEG confirmed the true diagnosis his mother had evolved a very complex diet which she was convinced had improved his behaviour and frequency of attacks. She was very reluctant to use anticonvulsant treatment instead of diets. There was a strong maternal family history of schizophrenia. The mother also believed that her son had reactive hypoglycaemia and administered 


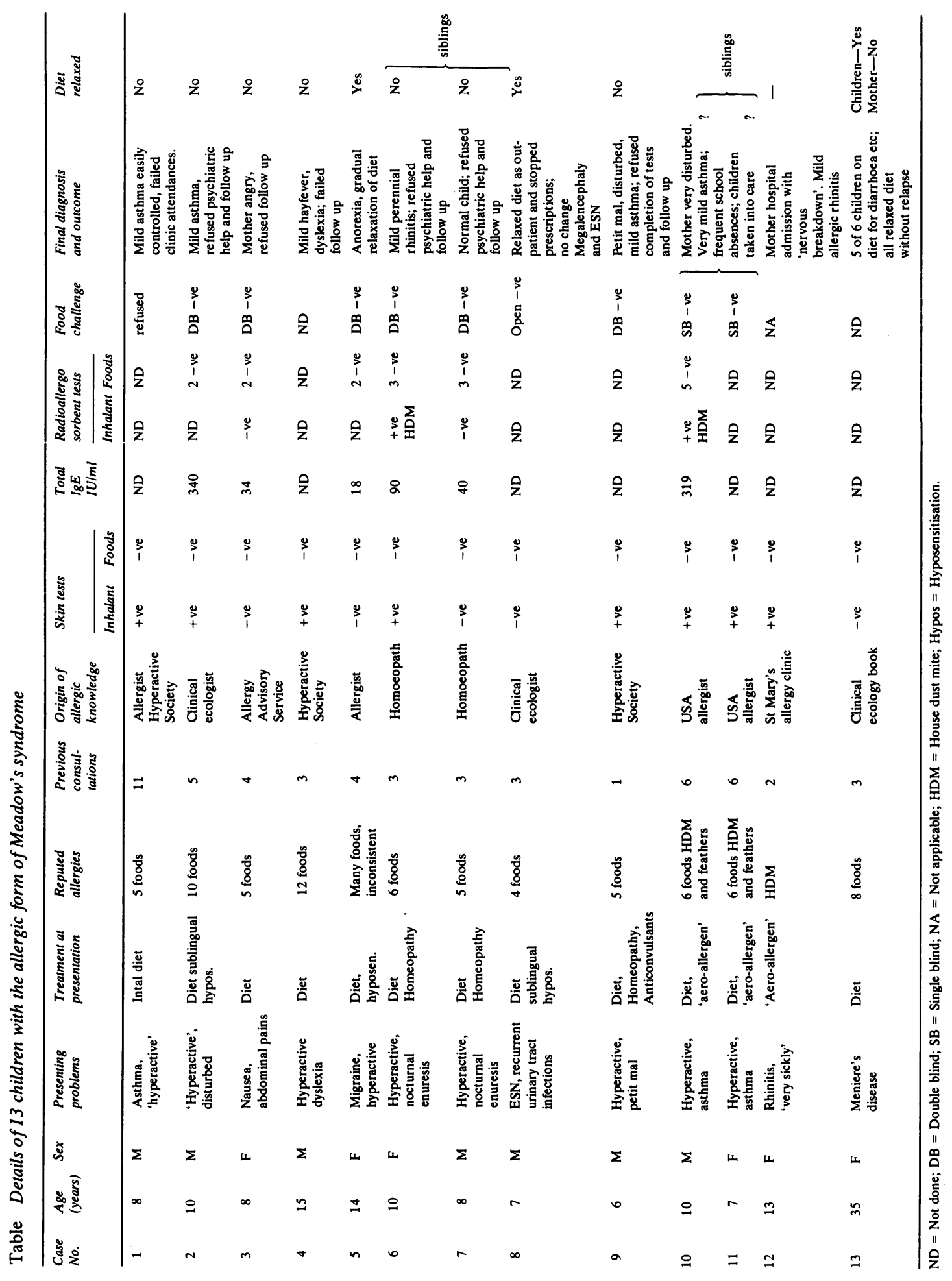


homoeopathic treatments. We could not establish any evidence of food intolerance on double blind challenges and he had normal blood sugars on several 24 hour profiles. Unfortunately his mother would not allow us to complete the studies and it has been left to the referring paediatrician to struggle with the child's management. Two extracts from the mother's very lengthy letters to us illustrate her obsessions. 'I find it interesting that when we considered zinc deficiency, copper overload 18 months ago ... when we altered the zinc/copper balance at the time there was a dramatic change in his behaviour and we have never allowed the situation to become so severe again'. 'We wonder whether he is hypoglycaemic around $4 \mathrm{pm}$ ... when we are in shops, even small, fairly empty ones, at $4.30 \mathrm{pm}$ he begins to shout at the shopkeepers, threatening to chop them up, boil them in a pot etc, ... we wondered whether he is affected by fluorescent lights, although some shops don't have them'. Her final comment on leaving the ward was 'The trouble with you doctors is that you don't believe in fairies'.

Ten of the 17 children had mild atopic disorders and the same 8 had evidence of allergy with positive skin tests to inhalant allergens but not to food allergens. Double blind food challenges were performed in 6 children and all were negative, with inconsistent responses on the offending and control foods. Single blind challenges were done on two siblings in hospital when it was possible to administer foods and make objective observation without maternal interference. One child had foods reintroduced without reaction as an outpatient. Two mothers refused admission to hospital for challenge and subsequently failed to attend for clinic appointments. One of these has turned up in another paediatric clinic and had failed to gain satisfaction from a previous 10 consultants. Five children had some form of aberrant behaviour such as temper tantrums and excessive aggression while observed on the ward, but these outbursts were not related to ingestion of particular foods. Seven children had had excessive and inappropriate school absences.

Mothers. The 11 mothers of the 17 children manifested many common features that have characterised other cases of Meadow's syndrome. Eight were articulate and well educated. It was not possible to ascertain whether there was a discrepancy in intellect between mothers and fathers as the fathers were notable by their absence. Two mothers were unmarried, one divorced, and four were suffering considerable marital stress. Two mothers had severe psychiatric problems and 6 were considered, from observations made by ward staff, to have abnormal relationships with their child. Five were on diets themselves without any obvious justification and although they were not investigated we wonder whether they were manifesting minor forms of Munchausen syndrome themselves. All but two mothers had involved a large number of specialists in the management of their children, and many had previously had vehement disagreements with these consultants. Lengthy exchanges of correspondence with consultants was another characteristic feature in 8 mothers. Four were involved with fringe religions or spiritualism and three were very active in parent self help groups for asthma or allergy. Their information about allergy had been obtained in 7 from doctors (allergists, homoeopaths, or clinical ecologists), one had approached the Allergy Advisory Service with hair cuttings to ascertain an allergy diagnosis, and three had read various literature (two from the Hyperactivity Society and one a book by a clinical ecologist).

Ten of the mothers exhibited a limpet-like attachment to their children. They insisted on vetting every action of the child which inevitably included supervising their feeding very closely, but also extended to washing, toileting, and play. Many made copious notes of their child's behaviour that were often at variance with the ward staff's observations. This resulted in frequent disputes over entries in patient record charts and caused discord on the ward with staff and other parents.

One notable mother (of cases 6 and 7) created enormous difficulties by suggesting to other parents that their child had food allergy. Her recommendations of diets for the patients, their mothers, and ward staff were extensive and it has taken a considerable time to undo the damage. During the course of a particularly tense consultation she even suggested homoeopathic treatment and diets for one of us (JOW). She has recently submitted a claim for an attendance allowance making incorrect assertions on our diagnoses and recommendations.

Outcome. In only three children was the outcome totally satisfactory, with permanent relaxation of the dietary restrictions and continued follow up. Three children improved while away from their mothers-one was voluntarily fostered and the other two were placed in temporary care. In five children, all of whom had negative double blind challenges, the mothers were angered by the results and rejected all attempts to redirect the treatment approach. All subsequently failed to attend for outpatient appointments, although two mothers wrote long and complex letters explaining the reasons for non-attendance, thanking us for our efforts, and indicating that the diets were being maintained. The 
remaining two did not progress as far as inpatient assessment before failing follow up. To date there have been no instances of physical abuse, cot death, or other tragedies in any of the families presented.

Diagnosis. None of the children had objective evidence of food intolerance or allergy. The mother of case 8 , a child with megalencephaly who was educationally subnormal, had been persuaded to seek medical attention by a school teacher. A consultation with a clinical ecologist had resulted in the diagnosis of food allergy in both mother and child. When the treatment became too expensive the mother sought help from the allergy clinic. After very careful investigation she would not accept our recommendations. Quite fortunately, however, the child became ill during a challenge and the mother's conviction was shattered by finding that he had straightforward bacterial tonsillitis. She accepted that she had been misled by previous observations and relaxed his diet without further problems. One suspects that this mother was just exhibiting natural parental concern and responding to recommendations made and that this case should not be classified as Meadow's syndrome.

The remaining 16 children with non-existent food related problems were being moulded into invalids by their mothers. The effect was to create enormous dependence of the child on the mother. The mothers, in turn, appeared as devoted parents who were sacrificing their lives and finances to maintain their child's health. While they were not submitted to very extensive medical investigation, the whole scenario was very similar to that seen in the cases described by Meadow. ${ }^{1}$ Case 5 was an extreme example with very considerable weight loss due to the diet. A very lengthy admission with many hours of discussion with the mother resulted in a slight change in the mother's attitude and an improvement in her daughter. She has thrived subsequently and her mother has diverted her energies to another cause (organising an allergy group).

\section{Discussion}

The study of food allergy or intolerance is dogged by the absence of objective tests to validate the diagnosis. Because of this, suspicion of fabrication cannot be confirmed absolutely and there is no doubt that reactions to foods may sometimes result in unexpected and bizarre symptoms. We still rely heavily on clinical observation and challenge tests, preferably using Goldman's criteria, which require a reproducible reaction to three consecutive challenges with improvement on withdrawal of the offending food. ${ }^{4}$ This is very difficult to achieve, however, in clinical practice. The rigorous investigation programme may itself serve to convince parents that there is a pathological and perhaps allergic basis to their child's symptoms. Furthermore allergy is common and may be a fortuitous (from the mother's point of view) coexistent abnormality. Thus, the diagnosis of food allergy or intolerance often remains in doubt and leads many paediatricians to err on the side of colluding with the mother's interpretation of the problem. The cases described here are, however, sufficiently characteristic, and the lack of evidence to support a diagnosis sufficiently certain to warrant their categorisation as the allergic form of Meadow's syndrome.

It must be emphasised that most patients investigated for food intolerance or allergy have genuine adverse reactions to foods. We have continually been surprised by the variety and unexpected nature of responses to foods. There is no doubt that behaviour can be affected by allergic reactions, although the exact relation between the reaction and cerebral function is not clear. Our experience matches those of others, that mood alteration in relation to foods never occurs in isolation, but may be associated with other more obvious reactions such as diarrhoea, urticaria, eczema, etc. ${ }^{5}$

Concern about diet as a cause of disease has become one of the great preoccupations of our age. Natural parental anxiety may lead to the interpretation that a particular symptom is due to a food. Apparent resolution when the food is avoided may represent a placebo effect of resolving parental anxiety, or recovery from intercurrent infection, or a genuine food intolerance. The belief that a food is causing problems often arises during weaning, which coincides with rapid changes in an infant's physical responses and behaviour. Thus perfectly normal changes, such as occur in an infant's stool character or frequency may be wrongly considered abnormal and caused by food intolerance. The parental conviction is now further reinforced by extensive 'mass media' publicity of food allergy and the medical profession's inability to cater for the public concern. This has resulted in a proliferation of unorthodox approaches to the diagnosis and treatment of apparent food related disorders which are all too readily taken up by anxious parents. One leading allergist recently commented 'not since mesmerism and phrenology were in vogue in the nineteenth century has the public appeared so gullible and so vulnerable to fashionable nostrums' ${ }^{6}$ At its most extreme the preoccupation with food allergy and intolerance has lead to the lay concept of the 'total allergy syndrome' and in paediatrics to the 'allergic form of Meadow's syndrome'.

As stated by Lessof " it is not enough to castigate the concept and to ignore the patient'. Until we are 
more objective in our approach to food allergy and intolerance these cases will continue to arise. It is disturbing that in so many of the children we describe the myth of food allergy was perpetrated or perpetuated by doctors. This is very similar to the experience in an adult allergy clinic, ${ }^{7}$ where the patients with pseudo-allergy had evidence of emotional disturbance.

As with other forms of Meadow's syndrome the management is extremely difficult. These few cases stand out in our memories as having caused more problems and occupied more time than the genuine cases of food intolerance put together. Unfortunately confrontation, as suggested by Meadow, ${ }^{1}$ does not succeed in these cases because the problems are usually not severe enough to warrant enforcement of a new treatment regimen. We suspect that prevention is the most important approach. Sensible handling when these problems first arise may prevent mothers from developing an entrenched belief about the cause of their children's symptoms.

Thanks to Jocelyn Bagshaw for secretarial help.

\section{References}

1 Meadow R. Munchausen syndrome by proxy. Arch Dis Child 1982;57:92-8.

2 Anonymous. Meadow and Munchausen (Editorial). Lancet 1983;i:456.

3 Hathaway MJ, Warner JO. Compliance problems in the dietary management of eczema. Arch Dis Child 1983;58:463-4.

${ }^{4}$ Goldman AS, Anderson DW, Jr, Sellers WA, Saperstein S, Knicker WT, Halpern SR. Milk allergy. I. Oral challenge with milk and isolated milk proteins in allergic children. Pediatrics 1963;32:425-43.

5 Minford AMB, MacDonald A, Littlewood JM. Food intolerances and food allergy in children: a review of 68 cases. Arch Dis Child 1982;57:742-7.

6 Lessof MH. Total allergy-the passing of a fashion. Respiratory Disease in Practice 1983;1:4-6.

7 Pearson DJ, Rix KJB, Bentley SJ. Food allergy: how much in the mind? Lancet 1983;i:1259-61.

Correspondence to Dr J O Warner, Allergy Clinic, St Mary's Hospital, Praed Street, London W2.

Received 13 October 1983

\section{Commentary}

\section{S R MEADOW}

Department of Paediatricts and Child Health, St James's University Hospital, Leeds

Clinicians are familiar with parents who exaggerate their child's illness, are exceedingly anxious about minor symptoms, or who report symptoms and signs that are not apparent to others in the family and to other trained observers. The mothers have to be treated with understanding and skill in order to avoid over investigating the child; such families are a regular part of every paediatrician's work. The mother's behaviour becomes pathological and dangerous, however, when she resorts to extreme falsification or the fabrication of signs, and when the child's growth and development is hampered by excessive hospitalisation, investigations, or by restriction of activity and schooling by the mother.

Dr Warner selected 17 children from among several hundred allegedly allergic children who were found not to be allergic. The particular problem for these children, as study of the detail of the results and discussion sections of his paper shows, was the way in which extraordinary and unpleasant regimens were inflicted on the children because of the mothers' obsessions-for instance the school child who had to sleep on an upturned wardrobe wrapped in toilet paper and silver foil. Most of us would agree that that child was being abused fearfully.

The mothers probably did not fabricate signs, and most readers are likely to conclude that the mothers did not deliberately falsify the illness story or the symptoms. Unfortunately there is no sharp dividing line between deliberate falsification (malingering for conscious gain) and abnormal illness behaviour in which there is unconscious gain (hysterical behaviour). The end result for the child is the same, and can be both cruel and dangerous, regardless of the origin of the mother's behaviour.

It is interesting that in the 11 families neither the children nor their siblings seem to have suffered from other forms of child abuse, for there is an important link between parentally induced factitious illness and non-accidental poisoning, physical abuse, and sudden death. ${ }^{1}$

'Allergy' has been reported as an associated feature of many of the gross cases of Munchausen syndrome by proxy reported from several different countries in recent years. Of 71 British cases for whom I have details, 'allergy', though rarely the most worrying presentation, was an additional main presentation in 22. After a false story of seizures and after factitious bleeding it was the third most common presentation. ${ }^{1}$ The alleged allergies have been to a variety of substances-foods causing diarrhoea, chemicals causing behaviour problems, and chemicals, plasters, and procedures causing rashes. Allergy is one of several warning signals that may help the clinician to remember the possibility of factitious illness when dealing with a child who has prolonged, unexplained and complex illness which has been solved by neither extensive investigation nor treatment.

\section{Reference}

${ }^{1}$ Meadow R. Factitious illness $\rightarrow$ the hinterland of child abuse. Recent advances in paediatrics no 7. London: Churchill Livingstone, 1984:217-32. 